\title{
Probing the out-of-plane optical response of plasmonic nanostructures using spectroscopic ellipsometry
}

\author{
R. Verre, K. Fleischer, C. Smith, N. McAlinden, J. F. McGilp, and I. V. Shvets \\ Centre for Research on Adaptive Nanostructures and Nanodevices (CRANN), School of Physics, Dublin 2, Ireland
}

(Received 13 July 2011; published 29 August 2011)

\begin{abstract}
A simplified approach to investigate the out-of-plane response of plasmonic nanostructures using spectroscopic ellipsometry (SE) is presented. One-dimensional self-assembled arrays of Ag nanoparticles (NP's) were grown on stepped $\mathrm{Al}_{2} \mathrm{O}_{3}(0001)$, in ultrahigh vacuum, using deposition at a glancing angle. The SE response was measured with the plane of incidence aligned along, and across, the surface steps. From the raw data, an anisotropic surface excess function (ASEF) can be extracted, whose properties depend only on the dielectric function of the NP layer. Three resonances are clearly seen in the ASEF: two in-plane resonances, which correspond to the resonances measured using normal incidence reflection anisotropy spectroscopy, and the out-of-plane resonance. A dipole model is used to simulate the optical response of the NP layer, where the presence of the out-of-plane resonance provides an important additional constraint in developing the model.
\end{abstract}

DOI: 10.1103/PhysRevB.84.085440

PACS number(s): 61.46.-w, 78.67.-n, 81.07.-b

\section{INTRODUCTION}

The interaction between electromagnetic waves and conduction electrons in metallic nanoparticles (NP's) results in distinctive features, originating from localized surface plasmon (LSP) phenomena. ${ }^{1,2}$ LSP's allow tuning of the optical properties of patterned nanoscopic objects within the visible wavelength range, thus opening novel possibilities for optoelectronics, ${ }^{3}$ solar cells, ${ }^{4}$ or biological analysis. ${ }^{5}$

The resonant profile of plasmonic nanostructures is usually investigated in the far field with common spectrometers, and only the in-plane properties are addressed. ${ }^{6-8}$ Much less effort has been devoted to the characterization of the out-of-plane LSP resonances in plasmonic materials. ${ }^{9}$ This is due to the difficulty of measuring the out-of-plane component with common absorption spectrometer geometries. Spectroscopic ellipsometry (SE) can overcome this limitation as it monitors changes in the polarization state of a linearly polarized beam reflected at oblique angles of incidence. ${ }^{10,11}$ It is therefore a tool capable of investigating the full optical behavior of a plasmonic sample in the far field. ${ }^{12}$ Furthermore, SE can record the spectra with a better sensitivity than absorption spectroscopy, as only relative changes in the polarization state of the incoming light are measured. The major complication is that a model is required and the model compared with experiment to extract the key optical properties of the investigated material. An analysis of plasmonic structures is further complicated by the presence of the substrate, which modifies the overall optical response.

In this paper, we report how a quantity, the anisotropic surface excess function (ASEF), can be extracted directly from the measured signal. The great advantage of the ASEF is it is dominated by the optical properties of the plasmonic layer only. Thin plasmonic structures with in-plane anisotropy can be measured, the only limit to the approach being that the substrate must be optically isotropic. As amorphous materials, such as glass, or tetragonal unit-cell single-crystal structures satisfy this condition, the latter is not a major limitation for the approach. The full investigation of plasmonic resonances that this approach allows produces additional constraints in the modeling of the structures. Once the out-of-plane resonances are accessed, the parameters governing the out-of-plane optical response cannot be treated as fitting parameters, and modeling of the overall optical response is quite severely constrained.

Recently, we reported on the possibility of obtaining strongly coupled NP arrays on nanostructured templates ordered on the macroscale. ${ }^{13-15}$ The ability to tailor the surface morphologies and their optical in-plane resonances has been investigated with both polarized absorption spectroscopy in transmission and reflectance anisotropy spectroscopy (RAS), showing a strong in-plane dichroic response. Such structures are of considerable interest as they can be used for plasmon waveguides ${ }^{16,17}$ and enhancement effects. ${ }^{18-20}$

This paper discusses the growth of Ag NP on stepped $c$-plane $\mathrm{Al}_{2} \mathrm{O}_{3}$ substrates, characterized with both RAS and $\mathrm{SE}$. The equivalence of the two techniques in measuring the in-plane optical anisotropies of the plasmonic structures is demonstrated. The ASEF is extracted and a dipolar model is used to interpret the response.

\section{EXPERIMENT}

NP arrays were grown on a vicinal substrate, prepared by annealing in atmosphere. $\alpha-\mathrm{Al}_{2} \mathrm{O}_{3}$ (MTI Corporation, USA) with a $3^{\circ}$ off-cut from the low-index (0001) plane along the [1210] direction was chosen as a template for producing the stepped surface. The sample was cleaned in $\mathrm{HCl}$, rinsed, and sonicated in acetone, methanol, and high-grade propanol-2-ol, and immediately loaded into an alumina tube furnace. After annealing at $1450{ }^{\circ} \mathrm{C}$ for $24 \mathrm{~h}$, the surface was measured with atomic force microscopy (AFM) in tapping mode and showed the typical quasiperiodic pattern of facets and terraces previously reported. ${ }^{21}$ Axes are defined with respect to the vicinal substrate. The $x$ and $y$ axes lie in the optical plane, parallel and perpendicular to the steps, respectively, with the $z$ axis normal to the surface plane. The periodicity was measured to be $l_{y}=120 \pm 35 \mathrm{~nm}$. The template was subsequently loaded into an ultrahigh-vacuum chamber (base pressure $3 \times$ $10^{-8}$ mbar) and exposed for 20 min to a collimated Ag flux inclined at $6^{\circ}$ with respect to the average surface orientation, 


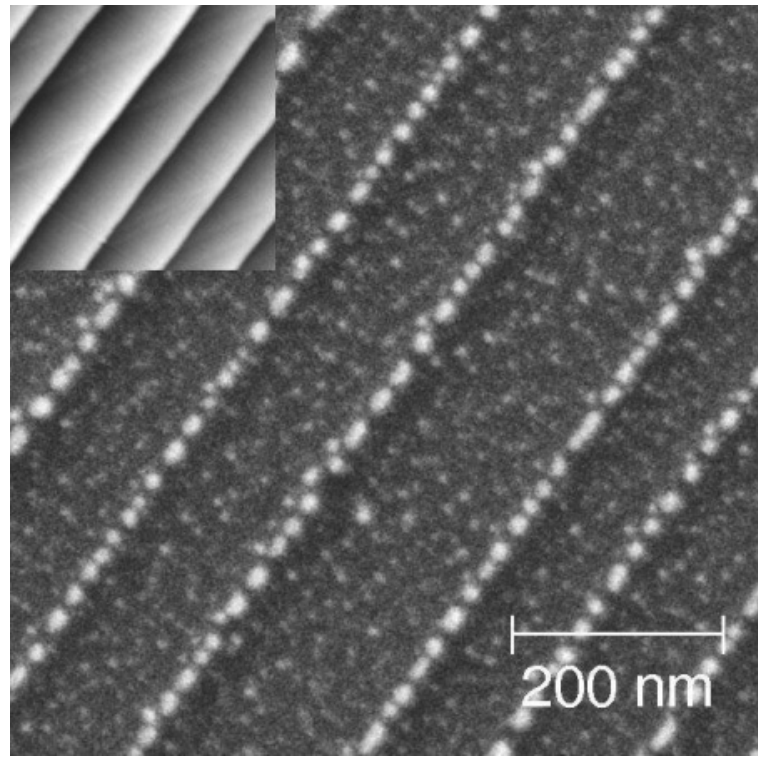

FIG. $1.700 \times 700 \mathrm{~nm}^{2}$ SEM image of $\mathrm{Ag} \mathrm{NP}$ arrays grown at a glancing angle of $6^{\circ}$ for $20 \mathrm{~min}$ on a faceted $\mathrm{Al}_{2} \mathrm{O}_{3}$ template. Inset: AFM image of the substrate prior to deposition.

and with a rate of deposition at normal incidence of $0.2 \mathrm{~nm} / \mathrm{min}$ measured by a quartz crystal balance. The surface was scanned with an ULTRA scanning electron microscope (SEM) (Zeiss, Germany) and the typical morphology is shown in Fig. 1.

The stepped surface is decorated by rows of elongated NP's aligned along the array axis $x$. Statistics of the semiaxes $R_{j}$, with $j=(x, y)$, the number of particles per unit area $N_{A}$, and the center-to-center interparticle distance $l_{j}$, have been extracted from the SEM images and are presented in Table I. The NP's have dimensions below the lithographic limit and an interparticle separation smaller than the average NP dimension, ensuring a strong coupling. As the deposition angle is larger than the off-cut angle, small clusters that are approximately five times smaller than the NP's on the facets appear deposited isotropically on the terraces. Their isotropic morphological distribution on the substrate will lead to a reduction in the RAS measured intensity, ${ }^{14}$ but, as no significant optical plasmonic response arises from such clusters, they will be disregarded in the modeling.

For optical characterization of the sample, both SE and RAS measurements were performed in reflection immediately after exposure of the sample to atmosphere. RAS measures the difference between the in-plane complex reflection coefficients along two orthogonal directions, averaged on the overall reflectance

$$
\frac{\Delta r}{r}=2 \frac{r_{x}-r_{y}}{r_{x}+r_{y}} .
$$

TABLE I. Morphology parameters of the sample measured with SEM and AFM.

\begin{tabular}{lcccc}
\hline \hline $\begin{array}{l}N_{A} \\
\left(\mathrm{NP}, \mathrm{s} / \mu \mathrm{m}^{-2}\right)\end{array}$ & $\begin{array}{c}l_{y} \\
(\mathrm{~nm})\end{array}$ & $\begin{array}{c}l_{x} \\
(\mathrm{~nm})\end{array}$ & $\begin{array}{c}R_{x} \\
(\mathrm{~nm})\end{array}$ & $\begin{array}{c}R_{y} \\
(\mathrm{~nm})\end{array}$ \\
\hline $347 \pm 133$ & $120 \pm 35$ & $24 \pm 6$ & $9.3 \pm 2.3$ & $7.7 \pm 1.4$ \\
\hline \hline
\end{tabular}

As RAS is measured at normal incidence, no information about the out-of-plane component is obtained. The RAS setup follows the Aspnes two-polarizer photoelastic modulator layout. ${ }^{14,22}$ Only the real part of the RAS signal has been recorded during the measurements in a range between 0.8 and $4.2 \mathrm{eV}$. The SE system is a Sopra Gesp 5 equipped with a Xe lamp. A microspot attachment produces a spot size of $\sim 300 \mu \mathrm{m}^{2}$, and measurements between 1.4 and $5 \mathrm{eV}$ were performed. Two measurements configurations were utilized: in the parallel measurement configuration $\left(\Phi=0^{\circ}\right)$, the plane of incidence was along the array chain, i.e., $E_{s} \| y$. In the perpendicular measurement configuration $\left(\Phi=90^{\circ}\right)$, after azimuthal rotation of the sample, $E_{s} \| x$. An angle of incidence of $\Theta=61^{\circ}$ has been chosen as it is close to the Brewster angle of the $\mathrm{Al}_{2} \mathrm{O}_{3}$ substrate, which minimizes the substrate contribution. Nomenclature and a sketch of the parallel measurement configuration are presented in Fig. 2

The pseudo bulk dielectric function $\left\langle\varepsilon_{j}\right\rangle$ of the sample can be determined directly from SE measurements, for both the parallel and perpendicular measurement configurations [see Figs. 3(a) and 3(b)]. The asymptotic behavior of the NP layer on top of the substrate follows that of the bare $\mathrm{Al}_{2} \mathrm{O}_{3}$ dielectric function. The plasmonic resonances appear in the visible range, departing from the optical behavior of the simple substrate only in the center of the measurement range. The real part $\operatorname{Re}\left[\left\langle\varepsilon_{j}\right\rangle\right]$ of the pseudo-dielectric function appears to be the simple sum of the substrate and of plasmonic peaks, whose position and intensity depend on the measurement configuration. A comparison of $\left\langle\varepsilon_{j}\right\rangle$ with the measured RAS spectra of Fig. 3(c) and similar previous results ${ }^{7,23,24}$ suggests that the positive peaks of Fig. 3(a) can be attributed to in-plane plasmonic resonances parallel ( $x$ resonance, $2.18 \mathrm{eV}$ in the present case) and perpendicular ( $y$ resonance, $3.24 \mathrm{eV}$ ) to the array axis.

The measured ellipsometry spectra can be compared to the RAS measurements of these structures that have been studied previously by us. ${ }^{14,15}$ The pseudo-dielectric function is the dielectric response of the solid phase of a two-phase system, consisting of the solid and ambient medium, where a sharp interface is assumed [see Fig. 2(c)]. For these samples, the solid phase comprises the substrate and NP layer. The standard anisotropic two-phase reflectance formula ${ }^{25}$ can be simply adapted to allow the RAS response to be extracted from SE measurements:

$$
\frac{\Delta r}{r}=\frac{1}{\sqrt{\langle\bar{\varepsilon}\rangle}} \frac{\left\langle\varepsilon_{x}\right\rangle-\left\langle\varepsilon_{y}\right\rangle}{\langle\bar{\varepsilon}\rangle-1},
$$

where $\langle\bar{\varepsilon}\rangle=\left(\left\langle\varepsilon_{x}\right\rangle+\left\langle\varepsilon_{y}\right\rangle\right) / 2$. The comparison between measured RAS and the RAS response extracted from ellipsometry is shown in Fig. 3(c). The agreement is very good, allowing for a small scaling factor associated with the different experimental geometries. The real parts of RAS and SE thus provide equivalent information on the in-plane reflection properties of Ag NP's and, as previously shown, ${ }^{14}$ they can also be related to polarized absorption spectroscopy measurements. In addition, the out-of-plane optical response is clearly revealed by the SE measurements. 


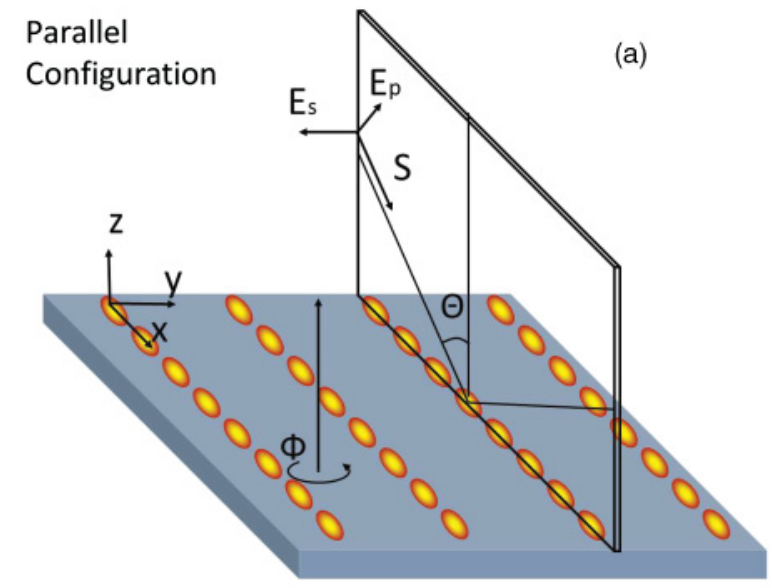

(b)

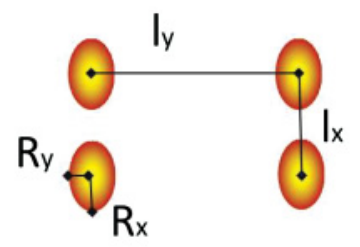

(c)
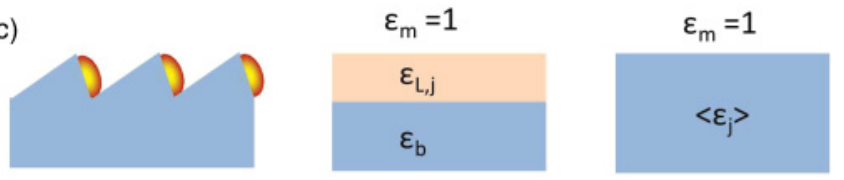

FIG. 2. (Color online) (a) Sketch of the SE measurement of $\mathrm{Ag}$ NP arrays in the parallel configuration (azimuthal angle $\Phi$ $=0^{\circ}$ ). The sample has three axes of symmetry, along the chain $(x)$, perpendicular to the chain $(y)$, and out-of-plane $(z)$. Light was incident (Poynting vector $\mathbf{S}$ ) at an angle of incidence $\Theta$ with respect to the normal, and the $s$ and $p$ components of the incident electric field are shown. In this configuration, the out-of-plane resonance and the in-plane resonance along the array axis are probed, while an azimuthal rotation of the substrate by $\Phi=90^{\circ}$ allows the resonance perpendicular to the array axis to be probed. (b) Morphological parameters. (c) Sample representation with the definitions used in the paper. In particular, the NP layer is considered to be anisotropic in the in-plane directions and homogeneous in the out-of-plane direction. An effective dielectric function $\varepsilon_{L, j}$ has been defined. Similarly, for the whole sample, considered as a single phase, the pseudo-dielectric function $\left\langle\varepsilon_{j}\right\rangle$ along the $j$ th direction is introduced.

\section{DISCUSSION}

Comparison of the SE and RAS results in Fig. 3 can be used to identify the $x$ and $y$ resonances, which then allow the minimum in $\operatorname{Re}\left[\left\langle\varepsilon_{j}\right\rangle\right]$ to be related to the $z$ resonance. The peaks in Fig. 3(a) show that $\operatorname{Re}\left[\left\langle\varepsilon_{j}\right\rangle\right]$ is associated with the absorptive response of the NP layer, and there appears to be a simple additivity of the substrate dielectric function $\varepsilon_{b}$ and NP layer dielectric function $\varepsilon_{L, j}$. Analytical modeling of the dielectric properties of single layers in a multilayered system, where there are multiple reflections between single layers, is not additive in general. ${ }^{10}$ Furthermore, in the present case, the system is far from being homogeneous at the interface due to the presence of steps and NP's. However, the latter problem is not significant regarding the optical response as the dimensions of the structures involved (surface roughness,
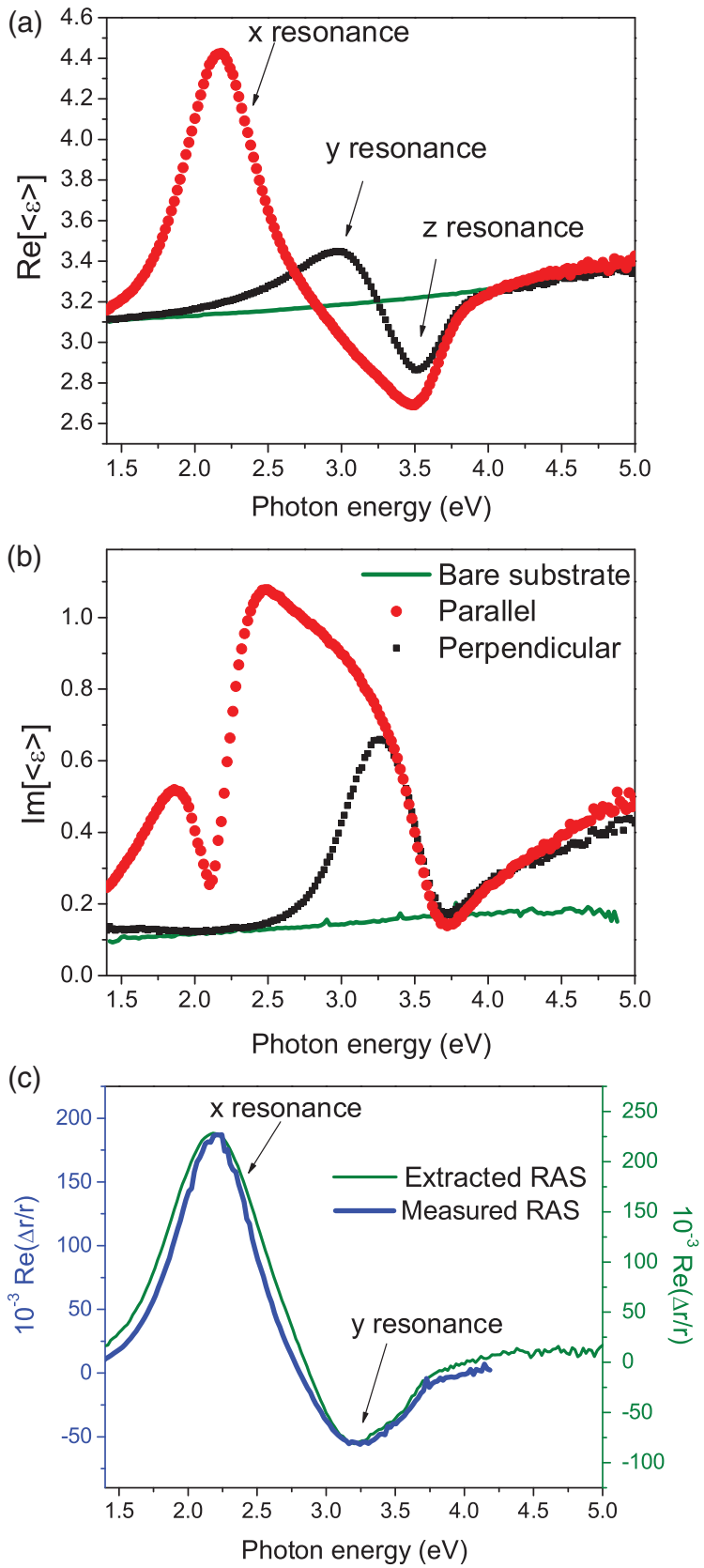

FIG. 3. (Color online) Extracted real (a) and imaginary (b) pseudo-dielectric function extracted from the ellipsometry measurements. (c) Comparison between the measured RAS (blue) and the in-plane response extracted from ellipsometry dielectric functions (green). The two spectra match very well, allowing for a small experimental scaling factor.

NP dimensions, and interparticle distances) are much smaller than the wavelength of the incident light. The quasistatic approximation hence holds and the cluster layer can be correctly regarded as a continuous medium with an effective anisotropic dielectric function $\varepsilon_{L, j} .{ }^{11}$

The additivity of bulk and NP response, as well as the relationship between $\operatorname{Re}\left[\left\langle\varepsilon_{j}\right\rangle\right]$ and $\varepsilon_{L, j}$, can be explained under the hypothesis of a thin anisotropic NP layer, bulk isotropy, and alignment of the optical and crystallographic axes. Previous 
work defining a surface excess function ${ }^{26-28}$ is adapted to make the anisotropic response explicit. The ASEF is given by

$$
\xi_{j}=\frac{i \lambda\left(\varepsilon_{b}-1\right)}{4 \pi \varepsilon_{b} \sqrt{\varepsilon_{b}-\sin ^{2} \Theta}}\left(\left\langle\varepsilon_{j}\right\rangle-\varepsilon_{b}\right),
$$

where $\lambda$ is the vacuum wavelength in nanometers, $\Theta$ is the angle of incidence, and, in the derivation, air was considered as the surrounding medium, with $\varepsilon_{m}=1$. The experimental results expressed in this way depend only on the response of the anisotropic layer, which can then be modeled using ${ }^{26}$

$$
\xi_{j}=d\left[\left(\Delta \varepsilon_{j}+\varepsilon_{b} \Delta \frac{1}{\varepsilon_{z}}\right)+\left(\frac{1}{\varepsilon_{b}}-\frac{\cos ^{2} \Theta}{\sin ^{2} \Theta}\right)\left(\Delta \varepsilon_{j}-\Delta \varepsilon_{k}\right)\right],
$$

where $j, k=(x, y)$. In Eq. (4), the coefficients are defined by

$$
\Delta \varepsilon_{j}=\varepsilon_{L, j}-\varepsilon_{b}, \quad \Delta \frac{1}{\varepsilon_{z}}=\frac{1}{\varepsilon_{L, z}}-\frac{1}{\varepsilon_{b}},
$$

and $d$ is the effective thickness of the NP layer. It can be seen that $\operatorname{Re}\left[\left\langle\varepsilon_{j}\right\rangle\right]$ depends on $\operatorname{Im}\left[\varepsilon_{L, j}\right]$ due to the imaginary factor in Eq. (3) and that the response is additive via the $\left\langle\varepsilon_{j}\right\rangle-\varepsilon_{b}$ term, provided $\varepsilon_{b}$ is real. The thin-film approximation explains the observations above and is valid provided

$$
d \ll \frac{\lambda}{4 \pi \sqrt{\varepsilon_{L, j}-\sin ^{2} \theta}} .
$$

In the spectral region of interest, it appears that this approximation can be usefully applied for films of average thickness less than $10 \mathrm{~nm}$, provided $\varepsilon_{L, j}$ is not too large. The average individual NP height has been estimated previously at $\sim 10 \mathrm{~nm}$ from transmission electron microscopy (TEM) studies, ${ }^{15}$ resulting in an average layer thickness of $1 \mathrm{~nm}$, for a filling factor of 0.1. For plasmonic structures such as these, the ASEF offers a simplified approach to interpreting SE measurements. However, the isotropy of the substrate assumed to derive Eq. (3) is not strictly correct in these experiments. $\mathrm{Al}_{2} \mathrm{O}_{3}$ is a well-known birefringent crystal, but we did not detect any change when the ASEF was calculated using ordinary or extraordinary refractive index values: ${ }^{29}$ the ordinary refractive index is assumed in the remainder of the paper. The imaginary part of the ASEF extracted in the present case is shown in Fig. 4(a). Three resonances can be observed and correspond, as previously mentioned, to resonances along the three main directions of the NP layer.

In order to reproduce the obtained signal, a dipolar model was used to calculate the effective dielectric function of the NP ensemble. The plasmonic layer was modeled as a collection of identical ellipsoids interacting by dipolar forces and placed in a rectangular lattice on the substrate. Under these assumptions, the in-plane and out-of-plane components become, respectively, ${ }^{15,30,31}$

$$
\begin{gathered}
\varepsilon_{L, j}=1+\frac{N \alpha_{j}}{1+\alpha_{j} \beta_{j}}, \\
\frac{1}{\varepsilon_{L, z}}=1-\frac{N \alpha_{z}}{1+\alpha_{z} \beta_{z}},
\end{gathered}
$$

where $N$ is the number of NP's per unit volume, $\alpha_{i}$ is the polarizability of an isolated $\mathrm{Ag}$ island along the $i=(x, y, z)$ direction, and $\beta_{i}$ is a coefficient that takes into account interparticle interaction and image-charge effects. The polarizability of an ellipsoidal particle in air is ${ }^{32}$

$$
\alpha_{i}=V \frac{\varepsilon-1}{1+L_{i}(\varepsilon-1)},
$$

with $L_{i}$ being the shape depolarization factor and $\varepsilon$ is the dielectric function of the metal. In the simulations, the dielectric function for $\mathrm{Ag}$ is assumed to be $\varepsilon_{i}=\varepsilon_{\infty}+$ $\omega_{p}^{2} /\left(\omega^{2}+i \omega \Gamma_{i}\right)$, with $\varepsilon_{\infty}=4.2$ and $\omega_{p}=8.85 \mathrm{eV}^{33} \mathrm{~A}$ correction in the bulk scattering rate $\Gamma_{i}=\Gamma_{\text {bulk }}+A v_{F} / R_{i}$ is also used, where $\Gamma_{\text {bulk }}=0.037 \mathrm{eV}$ is the bulk value, ${ }^{33}$ $v_{F}=1.4 \times 10^{6} \mathrm{~m} / \mathrm{s}$ is the Ag Fermi velocity, ${ }^{34}$ and $A$ is a constant of the order of unity. This correction is necessary in order to take into account the reduced relaxation time due to particle confinement and interface scattering. ${ }^{35} \mathrm{~A}$ value of $A=0.5$ was chosen to produce a peak width comparable to experiment. The interaction coefficient $\beta$ is defined by

$$
\beta_{j}=\frac{I}{4 \pi}\left(-F \times \sum_{m} C_{m, j}^{\prime}-\sum_{m} C_{m, j}\right)
$$

where $F=\frac{\varepsilon_{b}-1}{\varepsilon_{b}+1}$ and $C_{m}$ and $C_{m}^{\prime}$ are constants dependent on the position of the $m$ th NP dipole and the image-charge dipole, respectively, and the self-image term is included in the summation. ${ }^{30}$ It is well known that this simple dipole approach overestimates the interaction with the substrate, and thus the plasmon redshift, for flattened NP's. ${ }^{36,37}$ An analytical dipolar correction for an oblate spheroid ${ }^{15,37}$ is applied here, as the appropriate expressions are not available for flattened ellipsoids. Sharp edges of the NP's can produce multipolar resonances, but tarnishing of the outer layer on exposure to atmosphere tends to round these away and remove discrete multipolar features, as shown previously for tarnished Ag NP's. ${ }^{38}$ No multipolar features are observed in Fig. 3, but a modification of the position of the resonances cannot be excluded. It was found to be necessary to introduce a scaling factor $I$ in Eq. (10) to reproduce the measured peak splitting, revealing a limitation in applying this type of theoretical model to arrays of truncated ellipsoids. It is important to note the negative sign in Eq. (8) due to the boundary conditions of the out-of-plane component of the incident electric field in the Maxwell equations, ${ }^{39}$ which accounts for the minimum in the optical response in Fig. 3(a), being assigned effectively to the out-of-plane resonance.

The ASEF has been modeled using infinite arrays of identical ellipsoids with radii $R_{x}=9.3 \mathrm{~nm}, R_{y}=7.7 \mathrm{~nm}$, and $R_{z}=5 \mathrm{~nm}$, and center-to-center interparticle distances of $l_{x}=23 \mathrm{~nm}$ and $l_{y}=120 \mathrm{~nm}$, in accordance with the statistical analysis obtained with AFM and SEM (Table I). The effective layer thickness $d$ in Eq. (4) cannot be precisely determined and was set to $1 \mathrm{~nm}$ as discussed above. Figure 4(b) shows the simulated spectra with $I=1$ and 2.6. The presence of the scaling factor produces a further redshift of the peak positions, particularly the $x$ resonance position. With the parameters used, the agreement between experiment and theory is good if the NP dispersion, which will broaden the peaks and decrease their intensities, is considered. The shape of the extracted 

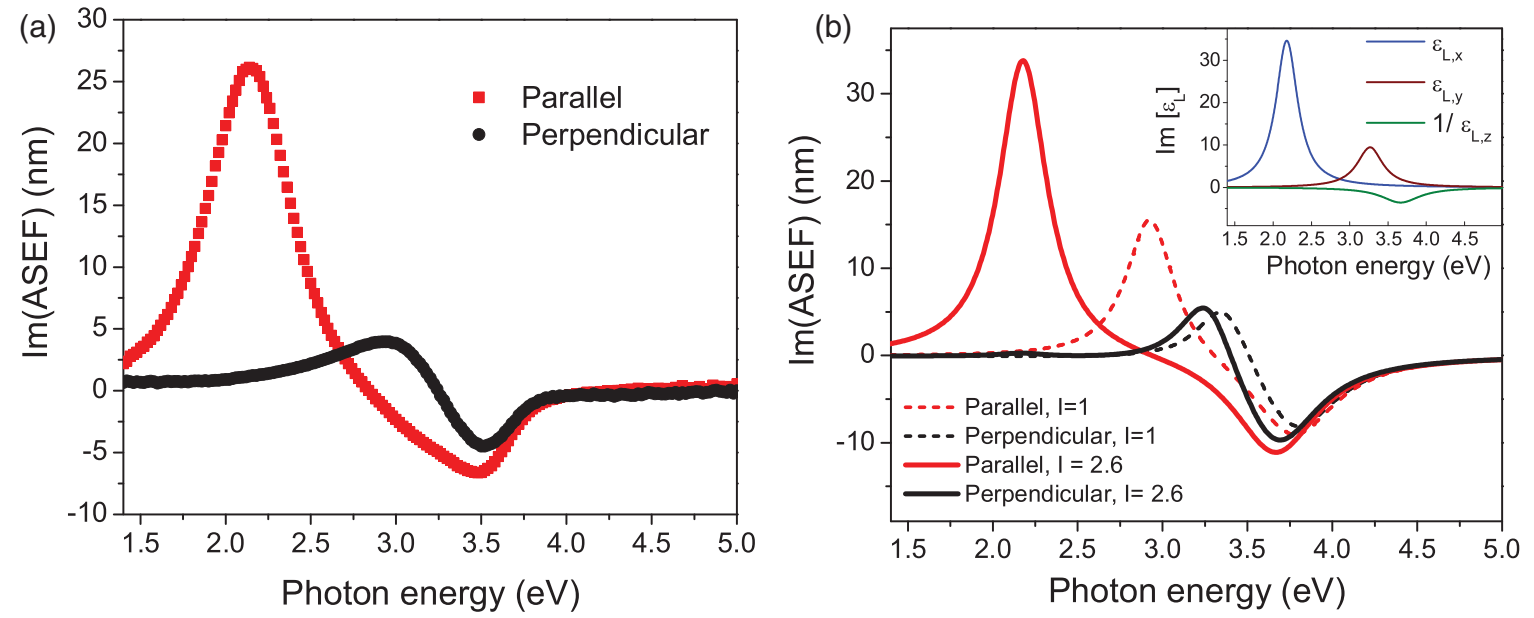

FIG. 4. (Color online) (a) Imaginary part of the ASEF obtained using Eq. (3). (b) Imaginary part of the simulated ASEF for different scaling factor $I$ of the interaction coefficients. The morphological parameters used were extracted from SEM analysis and are listed in Table I. Additional parameters used are $R_{z}=5 \mathrm{~nm}$, and $A=0.5$. A clear correspondence between experiment and theory can be seen. In the inset, the NP layer effective dielectric function $\varepsilon_{L}$ is shown for $I=2.6$.

ASEF is the same and the relative intensities match the experimental results. In particular, an increase can be noticed in the negative peak intensity of the ASEF with respect to $1 / \varepsilon_{z}$ (see inset) due to the $\varepsilon_{b} \Delta \frac{1}{\varepsilon_{z}}$ term in Eq. (4). Contributions to $\xi_{j}$ mainly come from $\varepsilon_{j}$ and $1 / \varepsilon_{z}$, as the second bracket in Eq. (4) is small for the particular experimental parameters used. The difference in the out-of-plane peak intensity between the calculated and measured ASEF in the parallel and perpendicular configurations is explained by the partial overlapping of the in-plane and out-of-plane peaks. It should be noted that the requirement to account for the position and intensity of all three in-plane and out-of-plane resonances places significant constraints on the development of appropriate models. It is clear that further work is needed in modeling the optical response of the arrays of truncated ellipsoids produced by these types of growth techniques.

The introduction of an ASEF thus allows the response of the plasmonic layer to be extracted directly from the SE measurements. This quantity can then be more simply modeled as the substrate does not have to be included. Similar results have been obtained on substrates with different periodicities and off-cuts, and it appears that this formalism may be generally applicable for thin plasmonic films.

\section{CONCLUSION}

Strongly dichroic self-assembled Ag NP arrays have been grown at a glancing angle of incidence on a stepped transparent template, and SE has been used to measure their full optical properties. RAS has also been measured and it is in agreement with the normal incidence response extracted directly from the SE measurements. For these thin layers it has been shown that an ASEF can be successfully extracted, which simplifies the development of theoretical models as the effect of the substrate is removed. In addition, the requirement to account simultaneously for the in-plane and out-of-plane resonant peak positions and intensities, extracted from the SE data, has revealed limitations in the current models of the optical response of arrays of truncated ellipsoids.

\section{ACKNOWLEDGMENTS}

We would like to thank R.G.S. Sofin for the useful suggestions. This work has been funded by the Science Foundation of Ireland, Contract No. 06/IN.1/I91, and conducted under the framework of the INSPIRE programme, funded by the Irish Government's Programme for Research in Third Level Institutions, Cycle 4, National Development Plan 2007-2013.

\footnotetext{
*rverre@tcd.ie

${ }^{1}$ U. Kreibig and M. Vollmer, Optical Properties of Metal Clusters (Springer, Berlin, 1957).

${ }^{2}$ L. Brongersma and P. Kik, Surface Plasmon Nanophotonics (Springer, Berlin, 2007).

${ }^{3}$ S. I. Bozhevolnyi, V. S. Volkov, E. Devaux, J.-Y. Laluet, and T. W. Ebbesen, Nature (London) 440, 508 (2006).

${ }^{4}$ S. Pillai, K. R. Catchpole, T. Trupke, and M. A. Green, J. Appl. Phys. 101, 093105 (2007).
}

${ }^{5}$ P. K. Jain, X. Huang, and I. El-Sayed, Plasmonics 2, 107 (2007).

${ }^{6}$ R. Lazzari, S. Roux, I. Simonsen, J. Jupille, D. Bedeaux, and J. Vlieger, Phys. Rev. B 65, 235424 (2002).

${ }^{7}$ E. Fort, C. Ricolleau, and J. Sau-Pueyo, Nano Lett. 3, 65 (2003).

${ }^{8}$ Q. Zhang, Y. Hu, S. Guo, J. Goebl, and Y. Yin, Nano Lett. 10, 5037 (2010).

${ }^{9}$ N. Large, L. Saviot, J. Margueritat, J. Gonzalo, C. N. Afonso, A. Arbouet, P. Langot, A. Mlayah, and J. Aizpurua, Nano Lett. 9, 3732 (2009). 
${ }^{10}$ R. Azzam and N. Bashara, Ellipsometry and Polarized Light (North Holland, Amsterdam, 1977).

${ }^{11}$ M. Losurdo, M. Bergmair, G. Bruno, D. Cattelan, C. Cobet, A. de Martino, K. Fleischer, Z. Dohcevic-Mitrovic, N. Esser, M. Galliet, R. Gajic, D. Hemzal, K. Hingerl, J. Humlicek, R. Ossikovski, Z. Popovic, and O. Saxl, J. Nanopart. Res. 11, 1521 (2009).

${ }^{12}$ E. S. Kooij, H. Wormeester, E. A. M. Brouwer, E. van Vroonhoven, A. van Silfhout, and B. Poelsema, Langmuir 18, 4401 (2002).

${ }^{13}$ F. Cuccureddu, S. Murphy, I. Shvets, M. Porcu, and H. W. Zandbergen, Nano Lett. 8, 3248 (2008).

${ }^{14}$ R. Verre, K. Fleischer, R. G. S. Sofin, N. McAlinden, J. F. McGilp, and I. V. Shvets, Phys. Rev. B 83, 125432 (2011).

${ }^{15}$ R. Verre, K. Fleischer, J. McGilp, D. Fox, G. Behan, H. Zhang, and I. Shvets (unpublished).

${ }^{16}$ M. Quinten, A. Leitner, J. R. Krenn, and F. R. Aussenegg, Opt. Lett. 23, 1331 (1998).

${ }^{17}$ B. Lamprecht, G. Schider, R. T. Lechner, H. Ditlbacher, J. R. Krenn, A. Leitner, and F. R. Aussenegg, Phys. Rev. Lett. 84, 4721 (2000).

${ }^{18}$ S. Zou and G. C. Schatz, Chem. Phys. Lett. 403, 62 (2005).

${ }^{19}$ S. Kim, J. Jin, Y.-J. Kim, I.-Y. Park, Y. Kim, and S.-W. Kim, Nature (London) 453, 757 (2008).

${ }^{20}$ A. Kinkhabwala, Z. Yu, S. Fan, Y. Avlasevich, K. Mullen, and W. E. Moerner, Nat. Phot. 3, 654 (2009).

${ }^{21}$ J. R. Heffelfinger, M. W. Bench, and C. B. Carter, Surf. Sci. 370, 168 (1997).

${ }^{22}$ D. E. Aspnes, J. Vac. Sci. Technol. B 3, 1018 (1985).

${ }^{23}$ S. Camelio, D. Babonneau, D. Lantiat, and L. Simonot, Europhys. Lett. 79, 47002 (2007).
${ }^{24}$ A. Toma, D. Chiappe, D. Massabo, C. Boragno, and F. B. de Mongeot, Appl. Phys. Lett. 93, 163104 (2008).

${ }^{25}$ D. E. Aspnes, J. Opt. Soc. Am. 63, 1380 (1973), Eq. (2.6).

${ }^{26}$ M. Kelly, S. Zollner, and M. Cardona, Surf. Sci. 285, 282 (1993).

${ }^{27}$ W. Plieth and K. Naegele, Surf. Sci. 64, 484 (1977).

${ }^{28}$ P. V. Santos, B. Koopmans, N. Esser, W. G. Schmidt, and F. Bechstedt, Phys. Rev. Lett. 77, 759 (1996).

${ }^{29}$ E. Palik, Handbook of Optical Constants of Solids III (Elsevier, Amsterdam, 1998).

${ }^{30}$ S. Camelio, D. Babonneau, D. Lantiat, L. Simonot, and F. Pailloux, Phys. Rev. B 80, 155434 (2009).

${ }^{31}$ T. Yamaguchi, S. Yoshida, and A. Kinbara, Thin Solid Films 21, 173 (1974).

${ }^{32} \mathrm{C}$. Bohren and D. Huffman, Absorption and Scattering of Light by Small Particles (Wiley, New York, 1983).

${ }^{33}$ C. J. Flaten and E. A. Stern, Phys. Rev. B 11, 638 (1975).

${ }^{34}$ J. W. Mitchell and R. G. Goodrich, Phys. Rev. B 32, 4969 (1985).

${ }^{35}$ H. Hövel, S. Fritz, A. Hilger, U. Kreibig, and M. Vollmer, Phys. Rev. B 48, 18178 (1993).

${ }^{36} \mathrm{D}$. Bedeaux and J. Vlieger, Optical Properties of Surfaces (Imperial College Press, London, 2001).

${ }^{37}$ M. Valamanesh, Y. Borensztein, C. Langlois, and E. Lacaze, J. Chem. Phys. C 115, 2914 (2011).

${ }^{38}$ J. M. Flores-Camacho, L. D. Sun, N. Saucedo Zeni, G. Weidlinger, M. Hohage, and P. Zeppenfeld, Phys. Rev. B 78, 075416 (2008).

${ }^{39}$ T. Yamaguchi, S. Yoshida, and A. Kinbara, Thin Solid Films 18, 63 (1973). 